\title{
MR-guided vacuum-assisted breast biopsy of MRI-only lesions: a single center experience
}

\author{
Claudio Spick $^{1} \cdot$ Melanie Schernthaner ${ }^{1} \cdot$ Katja Pinker $^{1}$ • \\ Panagiotis Kapetas $^{1} \cdot$ Maria Bernathova $^{1} \cdot$ Stephan H. Polanec $^{1} \cdot$ Hubert Bickel $^{1}$. \\ Georg J. Wengert ${ }^{1}$ - Margaretha Rudas ${ }^{2} \cdot$ Thomas H. Helbich $^{1}$ - Pascal A. Baltzer ${ }^{1}$
}

Received: 7 October 2015 / Revised: 20 January 2016 / Accepted: 1 February 2016 / Published online: 16 March 2016

(C) The Author(s) 2016. This article is published with open access at Springerlink.com

\begin{abstract}
Purpose The purpose of this study was to compare three different biopsy devices on false-negative and underestimation rates in MR-guided, vacuum-assisted breast biopsy (VABB) of MRI-only lesions.

Methods This retrospective, single-center study was IRB-approved. Informed consent was waived. 467 consecutive patients underwent 487 MR-guided VABB using three different 8-10-gauge-VABB devices (Atec-9-gauge,A; Mammotome8-gauge,M; Vacora-10-gauge,V). VABB data (lesion-type, size, biopsy device, histopathology) were compared to final diagnosis (surgery, $n=210$ and follow-up, $n=277$ ). Chisquare, and Kruskal-Wallis tests were applied. $P$ values $<0.05$ were considered significant.

Results Final diagnosis was malignant in 104 (21.4\%), high risk in $64(13.1 \%)$ and benign in $319(65.5 \%)$ cases. Eleven of $328(3.4 \%)$ benign-rated lesions were false-negative (1/95, $1.1 \%, \mathrm{~A} ; 2 / 73,2.7 \%, \mathrm{M} ; 8 / 1605.0 \% \mathrm{~V} ; P=0.095)$. Eleven high-risk $(11 / 77,14.3 \%)$ lesions proved to be malignant ( $3 /$ $26,11.5 \% \mathrm{~A} ; 4 / 12,33.3 \% \mathrm{M} ; 4 / 39,10.3 \% \mathrm{~V} ; P=0.228)$. Five of $34(14.7 \%)$ DCIS were upgraded to invasive cancer $(2 / 15,13.3 \%, \mathrm{~A} ; 1 / 6,16.6 \% \mathrm{M} ; 2 / 13,15.3 \%, \mathrm{~V} ; P=0.977)$. Lesion size $(P=0.05)$ and type (mass vs. non-mass, $P=0.107$ ) did not differ significantly.
\end{abstract}

Thomas H. Helbich

thomas.helbich@meduniwien.ac.at

1 Department of Biomedical Imaging and Image-guided Therapy, Division of Molecular and Gender Imaging, Medical University of Vienna (AKH), Waehringer Gürtel 18-20, Floor 7F,

A-1090 Wien, Austria

2 Clinical Institute of Pathology, Medical University of Vienna (AKH), Waehringer-Guertel 18-20, A-1090 Wien, Austria
Conclusion MR-guided VABB is an accurate method for diagnosis of MRI-only lesions. No significant differences on false-negative and underestimation rates were observed between three different biopsy devices.

Key Points

- MR-guided VABB is an accurate procedure for the diagnosis of MRI-only lesions.

- Similar false-negative and underestimation rates allow all three different MR-guided VABB devices for clinical application.

- High-risk lesions should undergo surgery due to a substantial underestimation rate.

- Agreement between MR-guided VABB and final diagnosis (benign/malignant) was 95.5\% (465/487).

Keywords Breast cancer · MR imaging · MR-guided biopsy · Vacuum assisted breast biopsy $\cdot$ MRI-only lesions

\section{Introduction}

Contrast-enhanced magnetic resonance imaging (CE-MRI) is the most sensitive method for the detection of breast cancer [1, 2]. The high sensitivity leads to detection of lesions visible only on breast MRI. MRI-only lesions are defined as lesions that cannot be detected by other imaging modalities, including second-look (MRI directed) ultrasound [3]. In case of a suspicious MRI-only lesion, histological verification with MRguided VABB is mandatory $[4,5]$.

It has been shown that MR-guided vacuum-assisted breast biopsies (VABB) are a fast, safe, and accurate method that cause only minimal scarring and no consecutive breast deformity compared to surgical breast biopsy [6-20]. However, reported cancer detection, as well as false-negative and underestimation rates using MR-guided VABB, vary considerably among previously published studies [6-20]. These differences 
can only be partly explained by different study designs and patient populations. The influence of the MR-guided VABB device used, as well as the needle size, on false-negative and underestimation rates still remains unclear, as studies comparing individual devices have not yet been performed. To address this issue, a comparative study of several MRI-guided VABB devices using standardized conditions is warranted.

The purpose of this study was to compare three different biopsy devices on false-negative and underestimation rates in MR-guided VABB of MRI-only lesions.

\section{Materials and methods}

\section{Study design}

This retrospective single-center study was institutional review board (IRB)-approved and informed consent was waived. In our tertiary care breast cancer assessment center, we collected data from the institutional database for all consecutive women with non-palpable MRI breast lesions that were occult both on mammography and ultrasound, and for whom successful MRguided biopsy was performed due to suspicious (BI-RADS 4 or 5) findings on CE-MRI. MRI-only cases were included in which a final diagnosis had been established either by open surgery, in case of malignant and high-risk lesions, or imaging follow-up, in case of benign lesions. A minimum of 12 months imaging follow-up, to establish a standard of reference, has been applied $[21,22]$.

There were 467 consecutive patients (mean age 52, range 18-87 years) who underwent 487 MR-guided biopsies in our department during 11/2006 to 7/2013 and who were included in this study (Fig. 1).

\section{MR-guided biopsy}

All MR-guided biopsies were performed on a 1.5-Tesla (T) system (Magnetom Avanto, Siemens Medical Solutions ${ }^{\circledR}$, Erlangen, Germany) using a dedicated double breast imaging and intervention coil (InVivo, Philips, Netherlands). A short imaging protocol for lesion localization, consisting of a dynamic contrast-enhanced T1-weighted, spoiled gradient echo (fast low angle shot, FLASH-3D) sequence [repetition time/echo time (TR/TE) $11 / 4.76 \mathrm{~ms}$; spatial resolution $0.7 \times 0.7 \times 3 \mathrm{~mm}$; 64 slices; acquisition time $58 \mathrm{sec}]$ was acquired before and repeated once or twice after the intravenous (IV) application of $0.15 \mathrm{mmol} / \mathrm{kg}$ body weight of Gd-DOTA (Dotarem, Guerbet, France) with an automatic injector (Spectris, Medrad, Germany) at $3 \mathrm{ml} / \mathrm{s}$. Automatically scaled subtraction images were obtained by subtracting pre-contrast images from post-contrast images using the vendor-supplied software.

Intervention planning was performed without additional software by manually referencing the MRI lesion localization to that of an MRI-compatible marker with positive T1-weighted contrast. After obtaining written, informed consent for the procedure, vacuum-assisted biopsy using three different 8- to 10gauge devices (Vacora [V], Bard Biopsy Systems, Tampa, USA; Mammotome [M], Devicor Medical Products, Cincinnati, USA; or ATEC [A], Hologic, Indianapolis, USA) was performed by one of four experienced, board-certified radiologists (T.H.H., K.P, P.K., B.A.T.). The following needles were used for A (9-gauge Atec MRI needle, Hologic, Indianapolis, USA), M (8-gauge MRI needle, Devicor Medical Products, Cincinnati, USA), V (10-gauge MRI needle, Bard Biopsy Systems, Tampa, USA). The following markers were used for A (Atec TriMark, Hologic), M (Mammomark $3.5 \mathrm{~mm}$, Devicor Medical Products) and V (BiomarC $1 \times 5 \mathrm{~mm}$, Carbon Medical Technologies, MI, USA). M and A are MR-guided, console-based vacuum biopsy systems, whereas V is a hand-held vacuum biopsy system. All radiologists performed at least 20 MR-guided biopsies with each respective device. One radiologist who had performed more than 200 MR-guided biopsies in different institutions using different devices supervised the first 15 cases of the other 3 radiologists. The selection of each device was based solely on its availability during the study period and never on lesion type or needle size.

The FLASH-3D sequence above was repeated to verify needle positioning during the procedure. The number of samples obtained was 12-24, depending on lesion size and location [23]. The biopsy site was marked with MRI-compatible clip markers. To verify biopsy site and clip position and to exclude potential complications, such as major bleeding, a final FLASH-3D sequence was obtained. The results of histopathological analysis, which was performed by an experienced, board-certified breast pathologist (M.R.), were compared with the MR imaging results in interdisciplinary consensus. The B classification was applied for histological diagnosis [24]. In cases of discrepancy between imaging and histopathology, and in case of lesions with uncertain malignant potential (B3), open biopsy was performed after wire localization by boardcertified, experienced breast surgeons. In case of a benign finding, there was MR imaging follow-up of at least 12 months. Reasons for surgery on a benign VABB result were suspicion of poor targeting, e.g. epithelial hyperplasia without atypia as histological diagnosis on MR-guided VABB when a solid mass lesion was seen on MRI - such findings are deemed discrepant and usually undergo surgery in our department.

\section{Data analysis}

\section{Standard of reference}

MR-guided VABB data were obtained, including lesion type, size, biopsy device, and histopathology. The standard of reference final diagnosis (benign or malignant), was derived 


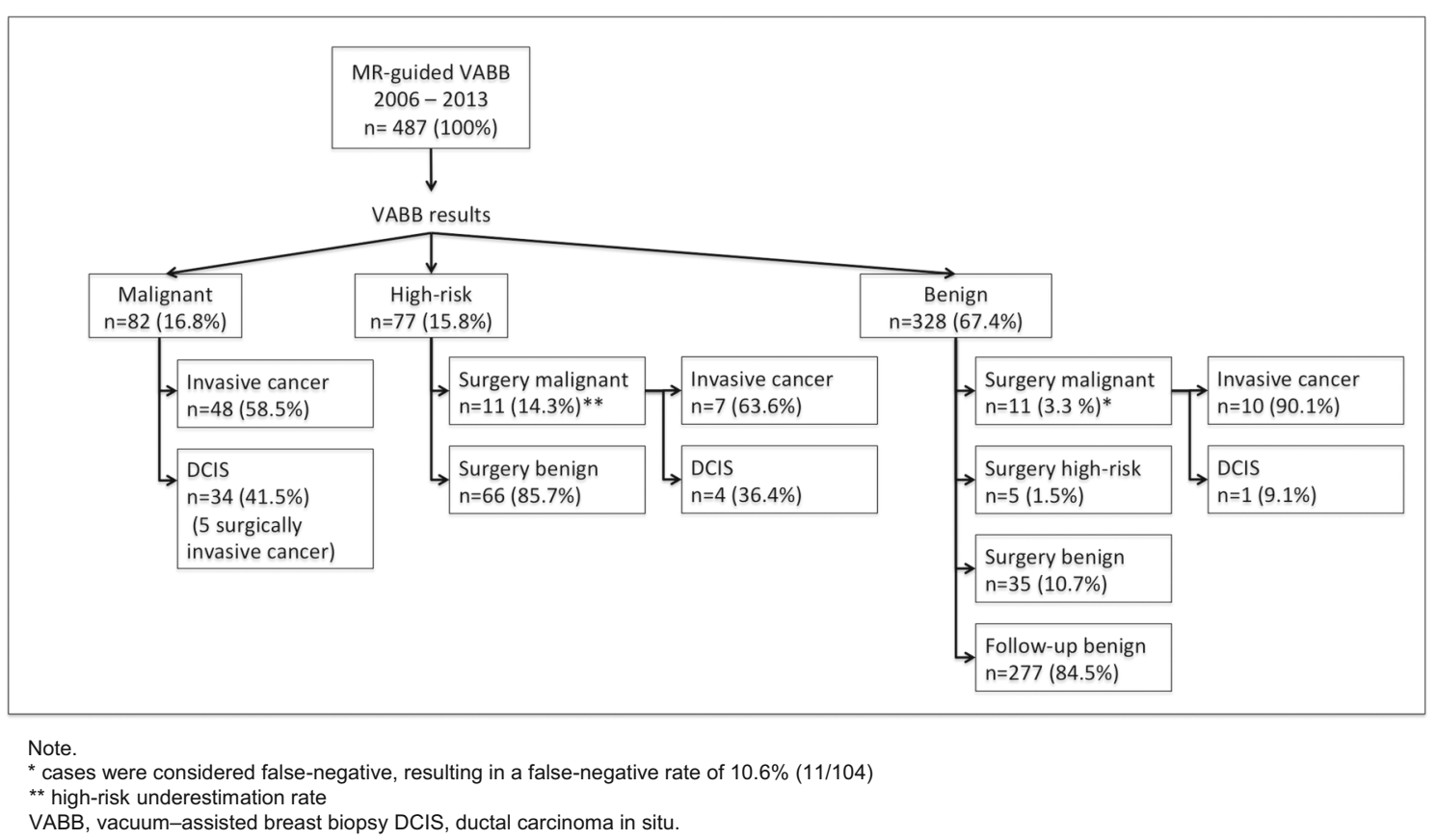

Fig. 1 Study design flowchart

from surgery ( $n=210$; histopathology subtype diagnosis: benign, high-risk, ductal carcinoma in situ [DCIS], invasive cancer [IC]) or imaging follow-up ( $n=277$ ) of at least 12 months. Data analysis followed the methodology established in a prior investigation [25].

\section{Association of MR-guided VABB results and standard of reference}

Agreement or discrepancies between MR-guided VABB results regarding final diagnosis (benign and malignant) and subtype diagnosis (benign, high-risk, DCIS, IC) were documented. A $\mathrm{VABB} /$ imaging discrepancy was defined as a discrepancy between imaging and VABB results. If, e.g. pathology described general tissue changes (fibrosis, unspecific epithelial hyperplasia) in case of a circumscribed enhancing lesion, this was deemed as not consistent with imaging findings. MR-guided VABB cases without a change of final diagnosis (benign and malignant) but with a different subtype diagnosis are reported separately.

\section{High-risk lesion underestimation rate}

High-risk lesions were atypical ductal hyperplasia (ADH), lobular carcinoma in situ (LCIS), papillary lesions (intraductal papilloma and papilloma with atypia), radial scar, and phyllodes tumour [25]. High-risk lesions that were diagnosed at MR-guided VABB, and in which a subsequent diagnosis of invasive cancer or DCIS lesion was made at surgical excision, were considered underestimates, after multidisciplinary review [26]. The high-risk lesion underestimation rate was defined as the number of these underestimated lesions divided by all high-risk lesions at MR-guided VABB histologic examination [27]. The final diagnosis (benign versus malignant) served as a reference standard for underestimation-rates. MR-guided VABB cases without a change of final diagnosis (benign and malignant) but with a different subtype diagnosis are reported separately.

\section{False-negative rate}

A false-negative result was considered a pathologically proven malignancy in which MR-guided VABB yielded benign findings. The false-negative rate was defined as the number of false-negative cases divided by the number of patients with cancer diagnosed.

\section{Statistical analysis}

Statistical analysis was performed using SPSS 20 (IBM, Chicago, USA) and Medcalc 12.7.5.0 (Mariakerke, Belgium). Descriptive statistics included calculation of the mean, standard deviation (SD), and $95 \%$ confidence intervals (95\% CI).

To assess the association of the different MR-guided VABB devices with underestimation and false-negative biopsy rates, a Chi-square test was used.

Continuous variables distribution was assessed by a Kolmogorov-Smirnoff test and compared by non parametric tests (Kruskal-Wallis) as the normal distribution was not verified. $P$ values of 0.05 or less were considered to indicate a statistically significant result. 


\section{Results}

\section{Characteristics of study population}

The median size of 487 MR-guided, VABB-proven breast lesions was $15.78 \mathrm{~mm}$ (range, 3-76 mm). Between three different MR-guided VABB devices, lesion size did not differ significantly $(P=0.05)$. The distribution rates among the 3 MR-guided VABB devices were as follows: 157/487 (32.2 \%, A); 101/487 (20.7\%, M); and 229/487 (47 \%, V). Patient age did not differ significantly among the three MRguided VABB devices $(P=0.112)$. Final diagnosis was malignant in $104(21.4 \%)$ and benign in $383(78.6 \%)$. The distribution of mass and non-mass lesions between the three MRguided VABB devices was: 122/229 were mass lesions in V $(53.3 \%) ; 68 / 101$ in $\mathrm{M}(67.3 \%)$; and $89 / 157$ in A (56.7\%; $P=0.107)$.

\section{MR-guided VABB results}

The histologic examination at MR-guided VABB yielded 48 of 487 (9.9\%) invasive carcinomas, 34 of 487 (7.0\%) DCIS lesions, 77 of 487 (15.8\%) high-risk lesions, and 328 of 487 $(67.4 \%)$ benign lesions. Agreement between MR-guided VABB results regarding exact final subtype diagnosis (benign, high-risk, DCIS, IC) was achieved in 448 of 487 (91.9\%; Table 1). In accordance with the standard of reference agreement between MR-guided VABB results and the final diagnosis (benign and malignant) was achieved in 465 of $487(95.5 \%)$ cases. MRI-guided VABB results and final diagnosis stratified by biopsy devices are shown in Table 2 .

Eighty-two of 104 (78.9\%) surgery-proven cancers were diagnosed by the initial MR-guided VABB. The distributions of malignant lesions between the 3 MR-guided VABB devices were as follows: 36/157 (22.9\%) A; 16/101 (15.8\%) M; 30/ $229(13.1 \%) \mathrm{V} ; P=0.103$. In five of 34 (14.7 \%) VABBproven DCIS lesions, diagnosis was upgraded to invasive cancer by surgery $(2 / 15,13.3 \%, \mathrm{~A} ; 1 / 6,16.6 \% \mathrm{M} ; 2 / 13$,
$15.3 \%, \mathrm{~V} ; P=0.977$; Tables 1 and 2). There were no falsepositive findings.

Thirty-five of $328(10.7 \%)$ benign lesions at MR-guided biopsy were confirmed by surgery (Table 1, Fig. 1). In five of $328(1.5 \%)$ lesions, MR-guided VABB diagnosed a benign lesion, and there was an up-grade based on surgery to a highrisk lesion, but not to cancer. All other 277 (84.5\%) benign MR-guided VABB were stable during a follow-up period of at least 12 months (median 44, range 12-92 months). Histopathological diagnoses are summarized in Table 3.

\section{High-risk lesion underestimation rates}

Eleven high-risk lesions were upgraded to cancer at surgery (seven invasive carcinomas, four DCIS lesions; Table 1, Figs. 1 and 2), resulting in a high-risk underestimation rate of $14.3 \%$ (11/77, $95 \%$ CI: 7.9-23.9\%). The distributions of all upgraded high-risk lesions among the 3 MR-guided VABB devices were as follows: $3 / 26(11.5 \%) \mathrm{A} ; 4 / 12(33.3 \%) \mathrm{M} ; 4 /$ $39(10.3 \%) \mathrm{V} ; P=0.228$ (Table 2). Seven $(9.1 \%, 7 / 77)$ highrisk lesions were confirmed as benign by surgery.

\section{False-negative rates}

Eleven of 328 (3.4\%) benign-rated lesions were false-negative, resulting in a negative predictive value (NPV) of $96.6 \%$ (317/328, $95 \%$ CI: 94.1-98.3\%). False-negative lesions were distributed among the three MR-guided VABB devices as follows: $1 / 95(1.1 \%) \mathrm{A} ; 2 / 73(2.7 \%) \mathrm{M} ; 8 / 160(5.0 \%) \mathrm{V}$; $P=0.095$. The resulting false-negative rate was $10.6 \%$ (11/ 104, $95 \%$ CI: $5.8-18.1 \% ; 1 / 40(2.5 \%)$ A; $2 / 22(9.1 \%)$ M; $8 /$ $42(19.0 \%) ; P=0.0611$; Tables 1 and 2, Fig. 1).

Eight of eleven $(66.7 \%)$ false-negative lesions were mass lesions. Characteristics of false-negatives are shown in Table 4. All eleven false-negative lesions were identified as discordant findings between imaging and MR-guided VABB results (Table 4, Fig. 3). The median size of the eleven falsenegative lesions was $11.5 \mathrm{~mm}$ (range, 5-60 mm). Numerical
Table 1 MR-guided VABB results and final diagnosis

\begin{tabular}{lcccrr}
\hline Final diagnosis & \multicolumn{2}{l}{ Biopsy results } & & \multirow{2}{*}{ Total } \\
\cline { 2 - 5 } & Benign & High-risk & DCIS & Invasive cancer & \\
\hline Benign & $312(97.8)$ & $7(2.2)$ & $0(0)$ & $0(0)$ & $319(100)$ \\
High-risk & $5(7.8)$ & $59(92.2)$ & $0(0)$ & $0(0)$ & $64(100)$ \\
DCIS & $1(2.9)$ & $4(11.8)$ & $29(85.3)$ & $0(0)$ & $34(100)$ \\
Invasive cancer & $10(14.3)$ & $7(10.0)$ & $5(7.1)$ & $48(68.6)$ & $70(100)$ \\
Total & $328(67.4)$ & $77(15.8)$ & $34(7.0)$ & $48(9.9)$ & $487(100)$ \\
\hline
\end{tabular}

Note: Numbers in parentheses are percentages

DCIS, ductal carcinoma in situ

VABB, vacuum assisted breast biopsy 
differences between the three MR-guided VABB devices did not reach significance (Table 2).

\section{Discussion}

The results of our study show no influence of three different biopsy devices on false-negative and underestimation rates in MR-guided VABB of MRI-only lesions. Our results confirm that MR-guided VABB is an accurate procedure for histopathologic verification of MRI-only lesions according to the standard of reference (surgery and follow-up). In accordance with the standard of reference, agreement between MR-guided VABB results and the final diagnosis (benign and malignant) was achieved in 465 of 487 (95.5\%).

Currently, there are several MR-guided VABB devices commercially available. The devices show minor variations in handling and needle size. MR-guided VABB has shown to be advantageous over large-core needle biopsy [28]. Several studies provide evidence that MR-guided VABB is an accurate method. In these studies, false-negative and underestimation rates ranged from $0-17 \%$ and $0-100 \%$, respectively [6-18, 20, 29]. The considerable differences seem to be caused by multiple factors, such as differences in investigating institutions, operators, VABB devices, and histopathological work-up.

We did not identify an influence of biopsy device on false-negative and underestimation rates among the three different MR-guided VABB devices. All MR-guided $\mathrm{VABB}$ in this comparative analysis were performed within one institution under standardized conditions by the same experienced radiologists, and the same experienced breast pathologist assessed the results. Therefore, we minimized possible factors that might have led to the previously reported differences in the performance of MR-guided VABB. In addition, our results did not confirm the findings from a previous study, which showed that an MR-guided, console-based, vacuum biopsy system differs from a hand-held vacuum biopsy system, and allows smaller lesions to be biopsied with higher operator confidence [16].

In our study, it was shown that accurate MR-guided VABB results are obtained regardless of the biopsy device used.

The false-negative rate of $10.6 \%$ (11 out of 104) in our study is acceptable compared to previously reported falsenegative rates ranging from $0-17 \%[6,7,13,16]$. All falsenegative findings were correctly identified due to radiohistopathological discordance. Consequently, no cancer was
Table 2 Correlation of MRguided VABB results and final diagnosis stratified by biopsy device

\begin{tabular}{|c|c|c|c|c|c|}
\hline \multirow[t]{2}{*}{ Final diagnosis } & \multicolumn{4}{|c|}{ Biopsy results } & \multirow[t]{2}{*}{ Total } \\
\hline & Benign & High-risk & DCIS & Invasive cancer & \\
\hline \multicolumn{6}{|l|}{ Vacora 10G } \\
\hline Benign & 148 (99.3) & $1(0.7)$ & $0(0)$ & $0(0)$ & 149 (100) \\
\hline High-risk & $4(10.5)$ & $34(89.5)$ & $0(0)$ & $0(0)$ & $38(100)$ \\
\hline DCIS & $1(6.7)$ & $3(20.0)$ & $11(73.3)$ & $0(0)$ & $15(100)$ \\
\hline Invasive cancer & 7 (25.9) & $1(3.7)$ & $2(7.4)$ & $17(63.0)$ & $27(100)$ \\
\hline Total & $160(69.9)$ & $39(17.0)$ & $13(5.7)$ & $17(7.4)$ & $229(100)$ \\
\hline \multicolumn{6}{|l|}{ Mammotome $8 \mathrm{G}$} \\
\hline Benign & 70 (98.6) & $1(1.4)$ & $0(0)$ & $0(0)$ & $71(100)$ \\
\hline High-risk & $1(12.5)$ & $7(87.5)$ & $0(0)$ & $0(0)$ & $8(100)$ \\
\hline DCIS & $0(0)$ & $1(16.7)$ & $5(83.3)$ & $0(0)$ & $6(100)$ \\
\hline Invasive cancer & $2(12.5)$ & $3(18.8)$ & $1(6.3)$ & $10(62.5)$ & $16(100)$ \\
\hline Total & $73(72.3)$ & $12(11.9)$ & $6(5.9)$ & $10(9.9)$ & $101(100)$ \\
\hline \multicolumn{6}{|l|}{ Atec 9G } \\
\hline Benign & 94 (94.9) & $5(5.1)$ & $0(0)$ & $0(0)$ & 99 (100) \\
\hline High-risk & $0(0)$ & $18(100)$ & $0(0)$ & $0(0)$ & $18(100)$ \\
\hline DCIS & $0(0)$ & $0(0)$ & $13(100)$ & $0(0)$ & $13(100)$ \\
\hline Invasive cancer & $1(3.7)$ & $3(11.1)$ & $2(7.4)$ & $21(77.8)$ & $27(100)$ \\
\hline Total & $95(60.5)$ & $26(16.6)$ & $15(9.6)$ & $21(13.4)$ & 157(100) \\
\hline
\end{tabular}

Note: Numbers in parentheses are percentages

DCIS, ductal carcinoma in situ

G, gauge

VABB, vacuum assisted breast biopsy 
Table 3 Characteristics of 487 MR-guided VABB results compared with the final diagnosis

\begin{tabular}{lrrrrr}
\hline Final diagnosis & \multicolumn{2}{l}{ Biopsy results } & \multicolumn{2}{c}{ Total } \\
\cline { 2 - 5 } & Benign & High-risk & DCIS & Invasive cancer & \\
\hline Malignant & 11 & 11 & 34 & 48 & 104 \\
High-risk lesion & 5 & 59 & 0 & 0 & 64 \\
Fibroadenoma & 19 & 0 & 0 & 0 & 19 \\
Scar & 7 & 0 & 0 & 0 & 7 \\
Fibrocystic disease & 158 & 3 & 0 & 0 & 161 \\
Inflammation & 11 & 0 & 0 & 0 & 11 \\
Unspecific & 60 & 0 & 0 & 0 & 60 \\
FAH & 31 & 1 & 0 & 0 & 32 \\
PASH & 14 & 0 & 0 & 0 & 14 \\
Papilloma* & 7 & 3 & 0 & 0 & 10 \\
Lymph node & 5 & 0 & 0 & 0 & 5 \\
Total & 328 & 77 & 34 & 48 & 487 \\
\hline
\end{tabular}

Note: Numbers are numeric

* solitary papilloma without atypia

DCIS, ductal carcinoma in situ

PASH, pseudoangiomatous stromal hyperplasia

$\mathrm{FAH}$, fibroadenomatoid hyperplasia

VABB, vacuum assisted breast biopsy

missed and patients were treated in appropriate time frames. This underlines the importance of regular multidisciplinary conferences to discuss image-guided biopsy findings and to avoid false-negative results. Importantly, MR-guided VABB showed a high NPV of $96.6 \%$.

MR-guided VABB revealed high-risk lesions in $16 \%$ (77 out of 487) of the biopsied lesions. In previous studies, there were varied reported underestimation rates, up to $100 \%$ [6-8, 11-14, 17-19]. The underestimation rate in our study is well within the lower range of reported underestimation rates in MR-guided VABB, ranging from 13-50\% [20]. Nevertheless, our results highlight the necessity for surgical biopsy of high-risk lesions revealed by MR-guided VABB to avoid missing breast cancer.

Although in agreement with previously published results, the malignancy rate in the current study was rather low. This can be explained by the composition of our patient collective in our tertiary care breast cancer assessment center. Patients either had an imaging abnormality when they presented for assessment or were high-risk patients who presented for screening. In screening, the prevalence of malignancy is low, leading to a proportionally higher number of false-positive findings. Furthermore, second-look (MRI-directed) ultrasound was subsequently performed in our department to localize MRI-detected lesions. In cases of successful secondlook ultrasound, further workup can then be performed under ultrasound guidance. Ultrasound-guided biopsies are, in general, desirable, as they are less costly, readily available, and more comfortable for the patient $[2,25]$. A recent metaanalysis on the utility of second-look ultrasound demonstrated
Fig. 2 A 44-year-old woman with a suspicious, segmental nonmass lesion classified as BIRADS 4. a: Pre-contrast scan showing the biopsy marker in its basic position. b: early contrastenhanced T1-weighted subtraction depicting a heterogeneous segmental non-mass lesion. c: control scan immediately before MRI-guided VABB with the biopsy marker indicating a correct positioning. d: subtraction corresponding to $\mathbf{c}$. Histopathology revealed a high risk lesion $\mathrm{ADH}$, atypical ductal hyperplasia). The patient underwent subsequent surgery where small nests of DCIS G2 were identified
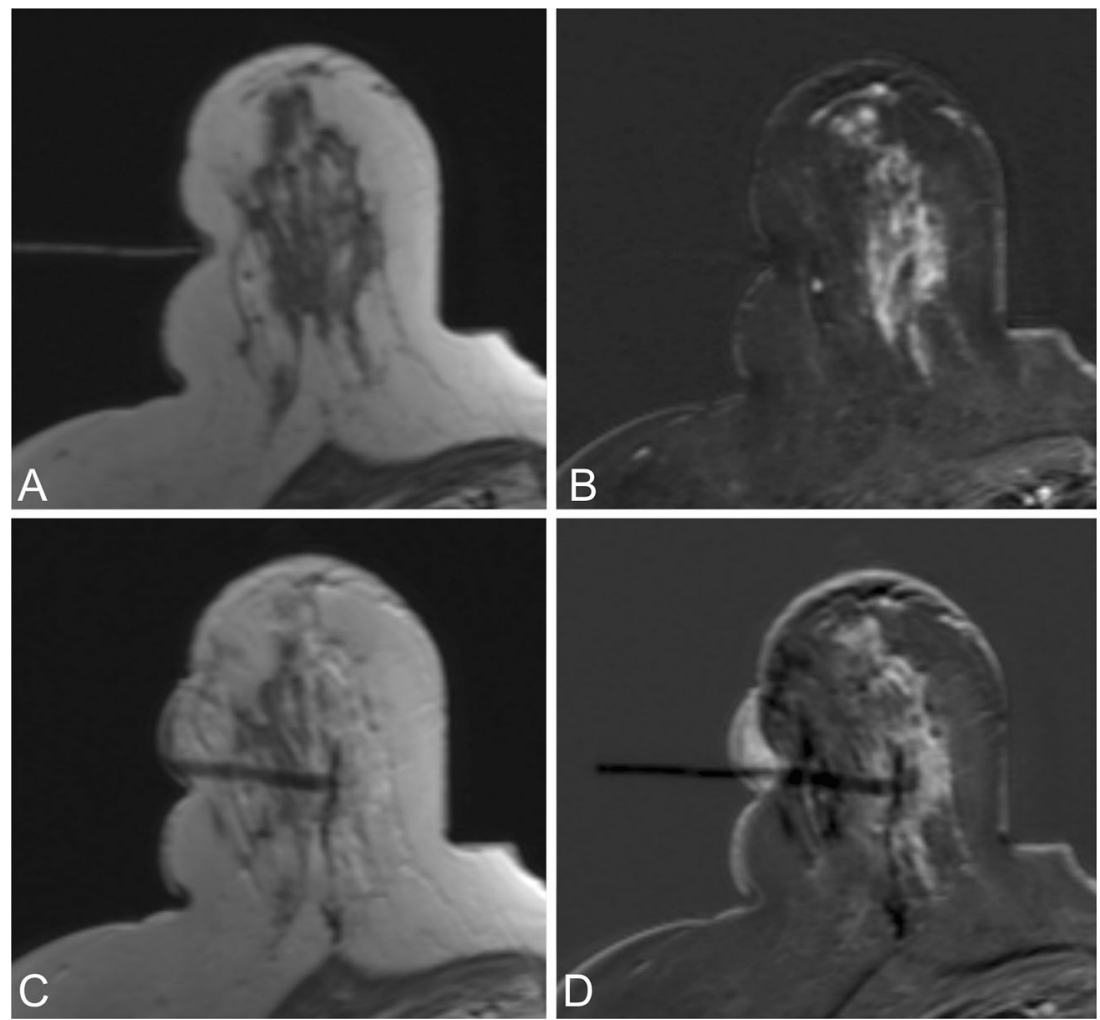
Table 4 Characteristics of 11 cases of false-negative MR-guided VABB results

\begin{tabular}{ll}
\hline & Total no. of lesions \\
\hline Lesion type: & \\
Mass & $8(66.7)$ \\
NME & $3(33.3)$ \\
Mass shape: & \\
Oval & $1(12.5)$ \\
Lobulated & $3(37.5)$ \\
Irregular & $4(50.0)$ \\
Mass margin: & \\
Circumscribed & $1(12.5)$ \\
Irregular/spiculated & $7(87.5)$ \\
Internal enhancement (mass): & $1(12.5)$ \\
Homogeneous & $1(12.5)$ \\
Dark internal septations & $6(75.0)$ \\
Heterogeneous & \\
NME distribution pattern: & $3(100)$ \\
Segmental & \\
Internal enhancement (NME): & $1(33.3)$ \\
Heterogeneous & $2(66.7)$ \\
Clumped & \\
Curve type: & $2(18.2)$ \\
Persistent & $6(54.5)$ \\
Wlateau & $3(27.3)$ \\
\hline & \\
Washout & \\
\hline
\end{tabular}

Note: Numbers in parentheses are percentages

DCIS, ductal carcinoma in situ

NME, non mass enhancement

VABB, vacuum assisted breast biopsy

that the majority of malignant lesions could be detected by second-look ultrasound. Nevertheless, even if second-look ultrasound is negative, there is a low, but significant, probability of malignancy. As we subsequently applied second-look ultrasound in our department, the rate of malignancy in this cross-sectional study was decreased compared to a setting in which second-look ultrasound is not used consistently [3].
Although MR-guided VABB is considered an accurate and safe method, CE-MRI of the breast leads to false-positive results in up to $74.1 \%$ [30]. This fact underlines the need for improved lesion differentiation between benign and malignant lesions to reduce unnecessary biopsies, and to improve the overall accuracy of CE-MRI of the breast. Recently, a study has reported that the addition of diffusion-weighted imaging (DWI) of the breast enables a reduction in false-positive, MR-guided biopsies by $>30 \%$ [30]. This is in good agreement with recently published results of multiparametric MRI of the breast, which resulted in a high diagnostic accuracy for MRI of breast tumours of [31, 32] and highlights the clinical usefulness of this technique in supporting a diagnosis.

Previous studies reported that MR-guided biopsy is not possible in up to $3 \%$ of cases $[7,13,18]$. In the current study, we did not experience that, due to strict selection criteria for MR-guided VABB, and second-look ultrasound was routinely performed. In all cases, imaging data were reviewed before MR-guided VABB was scheduled. If the successful performance of an MR-guided VABB was in doubt, i.e. close to the nipple or chest wall, a treatment decision for either MRguided VABB or MR-guided wire localization was reached in a multidisciplinary conference.

We provide the first empirical data on false negative and underestimation rates using three different VABB devices under the same clinical conditions, though this study is not free of limitations. The majority of benign findings had only imaging follow-up. However, in accordance with previous studies, we have used a minimum of 12 months of follow-up to establish a standard of reference [21, 22]. A further limitation of our study is that we did not report data on the intervention time of each device. Nevertheless, a previous study has already proven that console-based biopsy systems allow MRguided VABB in shorter intervention times, compared to hand-held devices [16]. Finally, numerical differences were in favor of the MR-guided, console-based vacuum biopsy system A (Table 2). However, they did not reach statistical significance when compared to $\mathrm{M}$ (console-based) and $\mathrm{V}$ (hand-held). This trend is in concordance with previous data showing higher accuracy for console-based (100\%) than
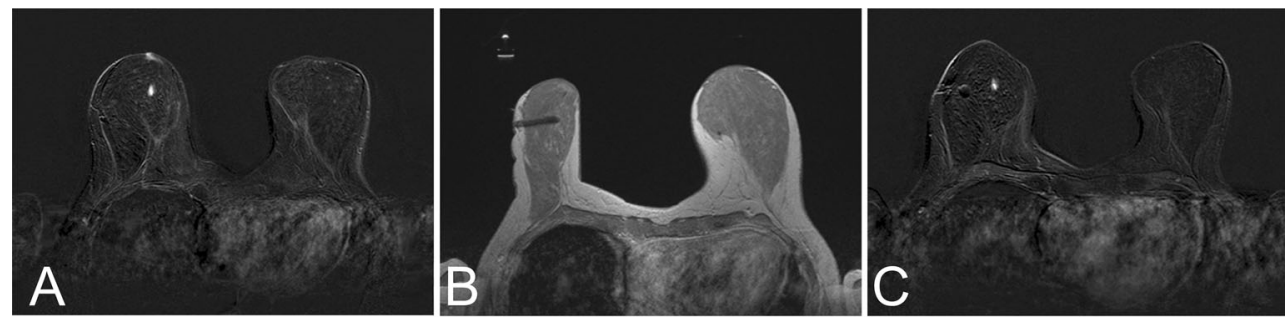

Fig. 3 a: A 41-year-old woman with an ill-defined mass lesion in the right breast classified BI-RADS 4. b: During intervention, the planing scan shows a seemingly correct position of the biopsy marker. Histopathology revealed benign breast tissue classified as B1. This case was rated discrepant as histology could not explain a solid lesion. c: Consequently, another MRI scan was performed after 2 weeks, showing the post-biopsy clip in a lateral position while the biopsied lesion was untouched. Re-biopsy revealed an IDC G2 
hand-held (98\%) systems [16]. In addition previously, the soft endpoint, operator confidence, appeared to be higher in console-based vacuum biopsy systems [16].

In conclusion, MR-guided VABB is an accurate procedure for the diagnosis of MRI-only lesions. There was no influence of the three different biopsy devices used on false-negative and underestimation rates. In case of radio-histopathological discordance (Fig. 3), repeat or surgical biopsy is recommended to avoid false-negative results. High-risk lesions should undergo subsequent surgery due to a substantial high-risk lesion underestimation rate.

Acknowledgments Open access funding provided by Medical University of Vienna. The scientific guarantor of this publication is Pascal A. Baltzer. This study was presented at the European Congress of Radiology (ECR) 2015 (SS 1002 - B-0767). The authors of this manuscript declare that the research reported in this article was supported by a seeding grant from Medicor/Hologic, Germany, Novomed, Austria and Guerbet, France. One of the authors has significant statistical expertise. Institutional review board approval was obtained. Written informed consent was waived by the Institutional Review Board. Methodology: retrospective, cross sectional study/observational, performed at one institution.

Open Access This article is distributed under the terms of the Creative Commons Attribution-NonCommercial 4.0 International License (http:// creativecommons.org/licenses/by-nc/4.0/), which permits any noncommercial use, distribution, and reproduction in any medium, provided you give appropriate credit to the original author(s) and the source, provide a link to the Creative Commons license, and indicate if changes were made.

\section{References}

1. Houssami N, Ciatto S, Macaskill P et al (2008) Accuracy and surgical impact of magnetic resonance imaging in breast cancer staging: systematic review and meta-analysis in detection of multifocal and multicentric cancer. J Clin Oncol 26:3248-3258

2. Warner E, Messersmith H, Causer P et al (2008) Systematic review: using magnetic resonance imaging to screen women at high risk for breast cancer. Ann Intern Med 148:671-679

3. Spick C, Baltzer PAT (2014) Diagnostic utility of second-look US for breast lesions identified at MR imaging: systematic review and meta-analysis. Radiology 273:401-409

4. Mann RM, Kuhl CK, Kinkel K, Boetes C (2008) Breast MRI: guidelines from the European society of breast imaging. Eur Radiol 18:1307-1318

5. Sardanelli F, Boetes C, Borisch B et al (2010) Magnetic resonance imaging of the breast: recommendations from the EUSOMA working group. Eur J Cancer 46:1296-1316

6. Rauch GM, Dogan BE, Smith TB et al (2012) Outcome analysis of 9-gauge MRI-guided vacuum-assisted core needle breast biopsies. AJR Am J Roentgenol 198:292-299

7. Imschweiler T, Haueisen H, Kampmann G et al (2014) MRI-guided vacuum-assisted breast biopsy: comparison with stereotactically guided and ultrasound-guided techniques. Eur Radiol 24:128-135
8. Perlet C, Heywang-Kobrunner SH, Heinig A et al (2006) Magnetic resonance-guided, vacuum-assisted breast biopsy: results from a European multicenter study of 538 lesions. Cancer 106:982-990

9. Heywang-Köbrunner SH, Heinig A, Schaumlöffel U et al (1999) MR-guided percutaneous excisional and incisional biopsy of breast lesions. Eur Radiol 9:1656-1665

10. Perlet C, Heinig A, Prat X et al (2002) Multicenter study for the evaluation of a dedicated biopsy device for MR-guided vacuum biopsy of the breast. Eur Radiol 12:1463-1470

11. Liberman L, Morris EA, Dershaw DD et al (2003) Fast MRI-guided vacuum-assisted breast biopsy: initial experience. AJR Am J Roentgenol 181:1283-1293

12. Lehman CD, Deperi ER, Peacock S et al (2005) Clinical experience with MRI-guided vacuum-assisted breast biopsy. AJR Am J Roentgenol 184:1782-1787

13. Liberman L, Bracero N, Morris E et al (2005) MRI-guided 9-gauge vacuum-assisted breast biopsy: initial clinical experience. AJR Am J Roentgenol 185:183-193

14. Orel SG, Rosen M, Mies C, Schnall MD (2006) MR imagingguided 9-gauge vacuum-assisted core-needle breast biopsy: initial experience. Radiology 238:54-61

15. Lee J-M, Kaplan JB, Murray MP et al (2007) Imaging histologic discordance at MRI-guided 9-gauge vacuum-assisted breast biopsy. AJR Am J Roentgenol 189:852-859

16. Schrading S, Simon B, Braun M et al (2010) MRI-guided breast biopsy: influence of choice of vacuum biopsy system on the mode of biopsy of MRI-only suspicious breast lesions. AJR Am J Roentgenol 194:1650-1657

17. Liberman L, Holland AE, Marjan D et al (2007) Underestimation of atypical ductal hyperplasia at MRI-guided 9-gauge vacuumassisted breast biopsy. AJR Am J Roentgenol 188:684-690

18. Malhaire C, El Khoury C, Thibault F et al (2010) Vacuum-assisted biopsies under MR guidance: results of 72 procedures. Eur Radiol 20:1554-1562

19. Han B-K, Schnall MD, Orel SG, Rosen M (2008) Outcome of MRI-guided breast biopsy. AJR Am J Roentgenol 191:1798-1804

20. Crystal P, Sadaf A, Bukhanov K et al (2011) High-risk lesions diagnosed at MRI-guided vacuum-assisted breast biopsy: can underestimation be predicted? Eur Radiol 21:582-589

21. Pisano ED, Gatsonis C, Hendrick E et al (2005) Diagnostic performance of digital versus film mammography for breast-cancer screening. N Engl J Med 353:1773-1783

22. Kelly KM, Dean J, Lee S-J, Comulada WS (2010) Breast cancer detection: radiologists' performance using mammography with and without automated whole-breast ultrasound. Eur Radiol 20:2557-2564

23. Floery D, Helbich TH (2006) MRI-Guided percutaneous biopsy of breast lesions: materials, techniques, success rates, and management in patients with suspected radiologic-pathologic mismatch. Magn Reson Imaging Clin N Am 14:411-425, viii

24. Perry N, Broders M, de Wolf C, Törnberg S, Schouten J (2001) EC working group on breast screening pathology. Quality assurance guidelines for pathology in mammography screening - non-operative diagnosis, chap. 6, in European guidelines for quality assurance in mammography screening, 3rd edn. Luxembourg, Office for Official Publications of the European Communities, pp 159-172

25. Schueller G, Jaromi S, Ponhold L et al (2008) US-guided 14-gauge core-needle breast biopsy: results of a validation study in 1352 cases. Radiology 248:406-413

26. Liberman L, Cohen MA, Dershaw DD et al (1995) Atypical ductal hyperplasia diagnosed at stereotaxic core biopsy of breast lesions: an indication for surgical biopsy. AJR Am J Roentgenol 164:11111113 
27. Verkooijen HM, Peeters PH, Buskens E et al (2000) Diagnostic accuracy of large-core needle biopsy for nonpalpable breast disease: a meta-analysis. Br J Cancer 82:1017-1021

28. Meeuwis C, Veltman J, van Hall HN et al (2012) MR-guided breast biopsy at 3T: diagnostic yield of large core needle biopsy compared with vacuum-assisted biopsy. Eur Radiol 22:341-349

29. Perlet C, Schneider P, Amaya B et al (2002) MR-Guided vacuum biopsy of 206 contrast-enhancing breast lesions. Röfo 174:88-95

30. Spick C, Pinker-Domenig K, Rudas M et al (2014) MRI-only lesions: application of diffusion-weighted imaging obviates unnecessary MR-guided breast biopsies. Eur Radiol 24:1204-1210
31. Pinker K, Bickel H, Helbich TH et al (2013) Combined contrastenhanced magnetic resonance and diffusion-weighted imaging reading adapted to the "breast imaging reporting and data system" for multiparametric 3-T imaging of breast lesions. Eur Radiol 23: 1791-1802

32. Pinker K, Bogner W, Baltzer P et al (2014) Improved diagnostic accuracy with multiparametric magnetic resonance imaging of the breast using dynamic contrast-enhanced magnetic resonance imaging, diffusion-weighted imaging, and 3-dimensional proton magnetic resonance spectroscopic imaging. Investig Radiol 49:421-430 